\title{
RADIATION CHARACTERISTICS OF A 2D PARABOLIC REFLECTOR ANTENNA EXCITED BY THE H-POLARIZED COMPLEX SOURCE
}

\author{
Taner Oguzer, Alexander I. Nosich ${ }^{*}$, Ayhan Altıntaş ${ }^{\text {** }}$ \\ Dokuz Eylül University, Electrical and Electronics Eng. Dept., Buca 35160, İzmir, Turkey \\ * Institute of Radio-Physics and Electronics, National Academy of Sciences, Kharkov 61085, Ukraine \\ ** Bilkent University, Electrical and Electronics Eng. Dept., Bilkent 06533 Ankara, TURKEY
}

\begin{abstract}
The aim of this paper is to obtain accurate reference data for the relatively large and realistic reflector antenna systems. Previously it has been done for a parabolic reflector antenna in E-case and now it is similarly performed for the H-polarization case. Directive primary feed is modeled by the complex source point method and the relative accuracy of the results is verified.
\end{abstract}

INTRODUCTION: Reflector antennas play an important role in the modern communication systems, and hence attract research efforts for efficient numerical analysis and optimization. Although twodimensional (2D) modeling relates only to cylindrical reflectors, it is still interesting in terms of advanced solutions and some practical applications. The scattering from a 2D reflector can be very accurately simulated by analytical-numerical techniques. One of them is the Riemann-Hilbert Problem (RHP) technique, which reduces the canonical circularly curved screen problem to a Fredholm $2^{\text {nd }}$ kind matrix equation after explicit inversion of the static part [1]. In [2], we had combined this solution with the complex source-point (CSP) method that enabled us to model realistic feed patterns in the both polarizations. However most of the realistic reflector surfaces are parabolic ones. This necessitates a modification of RHP technique to arbitrarily curved strips. In [3] we performed the analysis of 2D reflectors of conical-section profiles in the E-polarization case. Here we used a dynamic singularity extraction and inversion, as proposed in [4], to convert a logarithmic-singularity integral equation to the Fredholm 2-nd kind matrix equation. Furthermore the application of the FFT algorithms enabled us to treat electrically large geometries. In the H-polarization case, the basic integral equation has a strong singularity. It is worth to mention that similar problem was considered in [5]. Here, the integral equation was reformulated into a dual series equation (DSE) with trigonometric kernel. Then it was regularized by using a specialized method developed earlier for the equations with Jacobi polynomials. Later on, in [6] it was proposed to regularize the same DSE by the RHP technique. In the both studies, although a method was outlined, no numerical data were given for arbitrary profiles.

In this study, we regularize Electric Field Integral Equation (EFIE) obtained for the parabolic reflector antenna in the H-polarization case. EFIE is discretized by transforming it into the spectral domain and also written as DSE. To make regularization the static part is inverted analytically by the RHP technique since the static part of DSE constitutes a proper canonical form. Furthermore we can say that this is equivalent to the semi-inversion of the original IE containing hyper-type singularity in the kernel static part. In the details we used the FFT algorithm to perform computations of the double Fourier Series (FS) coefficients more rapidly. All these provide us to solve relatively large reflector antennas compared to the previous results with the semi-inversion techniques. This is the continuation of the work in $[2,3]$ and the results can be used to check the accuracy of the asymptotic and numerical techniques.

FORMULATION: The geometry of the problem can be defined so that infinitely thin PEC curved reflector surface having parabolic profile is illuminated by a directive feed (Figure 1). Parabola is a special case of an arbitrary profile. In the real space, the feed is located at the geometrical focus of the parabola. To perform regularization, parabolic reflector surface is completed to the closed contour $C$ by a circle having its origin on the $x$-axis. Its radius, $a$, is chosen so that, at the connection points, the slopes of the parabola and circle are matched. So the contour first derivatives are continuous and the discontinuities in the second derivatives are finite. The boundary value problem can be stated as the satisfaction of the Helmholtz equation, Sommerfeld radiation condition, and PEC boundary condition on reflector surface $M$. By usung the free-space Green's functions in 2D, i.e. $G^{A}{ }_{o}=G^{d s}=i / 4 H_{o}^{(I)}\left(k_{o}\left|r(\varphi)-r^{\prime}\left(\varphi^{\prime}\right)\right|\right)$, the tangential electric field can be written in terms of auxiliary vector and scalar potentials depending on tangential surface current $\left(\mathrm{J}_{\mathrm{t}}\right)$ and surface charge $(\rho)$ densities. 


$$
\vec{E}^{s c} \cdot \hat{t}=i \omega \vec{A} \cdot \hat{t}-\nabla \phi \cdot \hat{t}=i \omega \mu \int_{C}\left(\hat{t} \cdot \hat{t}^{\prime}\right) J_{t^{\prime}}\left(\vec{r}^{\prime}\right) G_{o}^{A}\left(\vec{r}, \vec{r}^{\prime}\right) d l^{\prime}-\hat{t} \cdot \nabla \int_{C}\left(\frac{1}{\varepsilon}\right) \rho\left(\vec{r}^{\prime}\right) G_{o}^{\phi}\left(\vec{r}, \vec{r}^{\prime}\right) d l^{\prime}
$$

Then by applying equation of charge conservation, i.e., $\nabla . \vec{J}=i \omega \rho$, and PEC boundary condition to the above equation and after manipulations the following integral equation is obtained:

$$
-E_{i}^{\prime n c}(s) \beta(s)=i \omega \mu \int_{0}^{2 \pi} J_{,}\left(s^{\prime}\right) \cos \left(\xi(s)-\xi\left(s^{\prime}\right)\right) \beta(s) \beta\left(s^{\prime}\right) G_{o}^{A}\left(s, s^{\prime}\right) d s^{\prime}-\left(\frac{1}{i \omega \varepsilon}\right) \frac{\partial}{\partial s} \int_{0}^{2 \pi}\left(\frac{\partial}{\partial s^{\prime}} J_{,}\left(s^{\prime}\right)\right) G_{o}^{\phi}\left(s, s^{\prime}\right) d s^{\prime}
$$

where $\beta(s)=r(s) / \cos (\gamma(s)), \xi(s)$ is the angle between the contour normal and the $x$-direction, and $\gamma(s)$ is the angle between the contour normal and the radial direction. Besides the closed contour $C$ is characterized by parametric equations $x=x(s), y=y(s), 0<s<2 \pi$ and the current density function $J_{t}$ is assumed zero on the part of $C$ complementary to $M$. The above IE is defined on $M$. On the slot part the current density is zero. The spectral versions of these two equations constitute a DSE. However a special care is needed for the Green's functions to find a regularized solution. Therefore we define the following new auxiliary regular functions,

$$
\begin{aligned}
& H\left(s, s^{\prime}\right)=H_{o}^{(1)}\left(k\left|\vec{r}(s)-\vec{r}\left(s^{\prime}\right)\right|\right)-H_{o}^{(1)}\left(2 k a\left|\sin \left(s-s^{\prime}\right) / 2\right|\right) \\
& G\left(s, s^{\prime}\right)=\cos \left(\xi(s)-\xi\left(s^{\prime}\right)\right) \beta(s) \beta\left(s^{\prime}\right) H_{o}^{(1)}\left(k\left|\vec{r}(s)-\vec{r}\left(s^{\prime}\right)\right|\right)-\beta^{2}(s) H_{o}^{(1)}\left(2 k a\left|\sin \left(s-s^{\prime}\right) / 2\right|\right)
\end{aligned}
$$

These functions $H\left(s, s^{\prime}\right)$ and $\mathrm{G}\left(s, s^{\prime}\right)$ have continuous first derivatives and second derivatives with respect to $\mathrm{s}$ and $\mathrm{s}$ ' and belong to $L_{2}$. For regularization these functions are expanded into double FS and the corresponding expansion coefficients rapidly decay like $O\left(1 /\left(\left.|n||m|\right|^{3 / 2 * \varepsilon}\right)\right.$. Then by using (3) and (4), DSE is written in certain canonical form and solved by RHP technique. The main problem in this process is an efficient computation of the double FS coefficients. We perform this by using FFT algorithms and exploit the fact that the second terms in (3) and (4) are expanded into FS analytically. All this provides us to handle larger problems than can be treated with earlier methods.

NUMERICAL RESULTS: Firstly the convergent nature of our algorithm is shown in Figure 2. The relative error in the computed surface current is $\max \left|x_{n}{ }^{N r{ }^{\prime \prime \prime}}-x_{n}{ }^{N r}\right| / \max \left|x_{n}{ }^{N / r}\right|$, that decreases quite rapidly with larger $N_{t r}$. It can be said that $0.1 \%$ accuracy can be obtained with $N_{t r}=2 k a+15$. Figure 3 presents a comparison of the radiation patterns obtained by the presented method and Physical Optics solution. It shows that excluding the back lobes the two methods show quite similar patterns but still some disappearances are observed in the penumbra region.

CONCLUSION: Two-dimensional parabolic reflector antenna illuminated with directive feed is solved by the analytical regularization in the H-polarization case. Presented numerical results have uniform 3digit accuracy although the algorithm can generate them with machine precision. Application of the FFT codes provides us to solve larger problems, and our data can be used as a reference to validate purely numerical or asymptotic methods.

\section{REFERENCES:}

[1] A.I. Nosich, "Green's function - dual series approach in wave scattering from combined resonant scatteres", in M. Hashimoto et al. (Eds.), Analytical and Numerical Methods in Electromagnetic Wave Theory, Tokyo: Science House, 1993, pp. 419-469.

[2] T. Oğuzer, A. Altıntaş A.I. Nosich, "Accurate simulation of reflector antennas by the complex source - dual series approach", IEEE Trans. Antennas Propagat., 1995, vol. AP-43, no 8, pp. 793-802.

[3] T. Oğuzer, A.l. Nosich and A. Altıntaş, "E-polarized beam scattering by an open cylindrical PEC strip having arbitrary conical-section profile", Microwave Optical Tech. Lett., 2001, vol. 31 no. 6, pp 480-484. 
[4] A.I. Nosich, S.V. Boriskina, "Economic and accurate in resonances solution to the wave scattering by arbitrary smooth dielectric cylinders, based on the canonical-shape inversion", IEEE Trans. Antennas Propagat., submitted in 2001.

[5] Y.A. Tuchkin, "Wave scattering by an open cylindrical screen of arbitrary profile with the Neumann boundary condition", Soviet Physics Doklady, vol. 32, pp. 213-214, 1987.

[6] V. Veremey, A. Poyedinchuk, "Two-dimensional scattering from a cavity-backed aperture with resonant loading", IEEE APS-URSI Int. Symp. Digest, Chicago, 1994, pp.1090-1093.

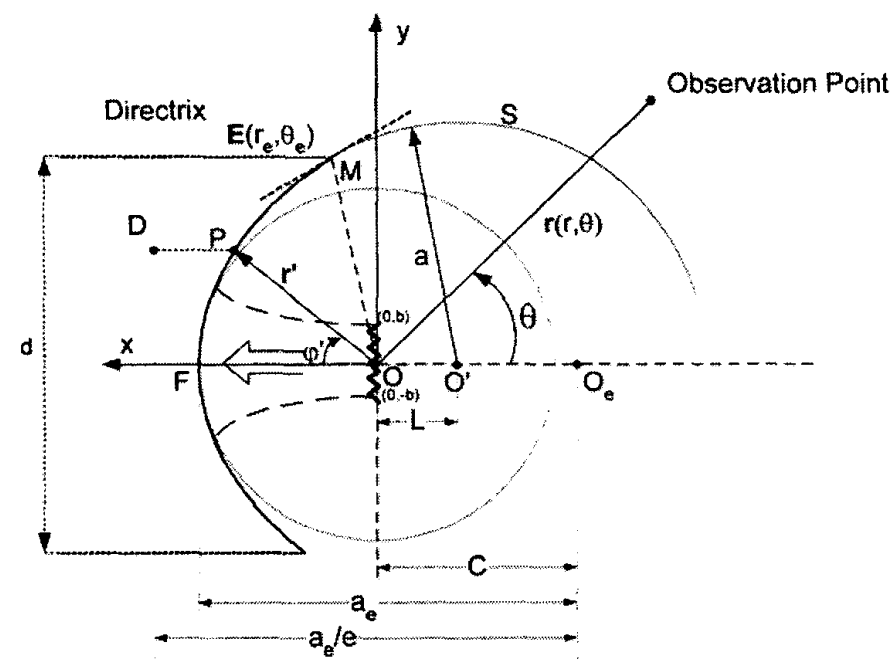

Figure 1: Geometry of 2D reflector antenna system

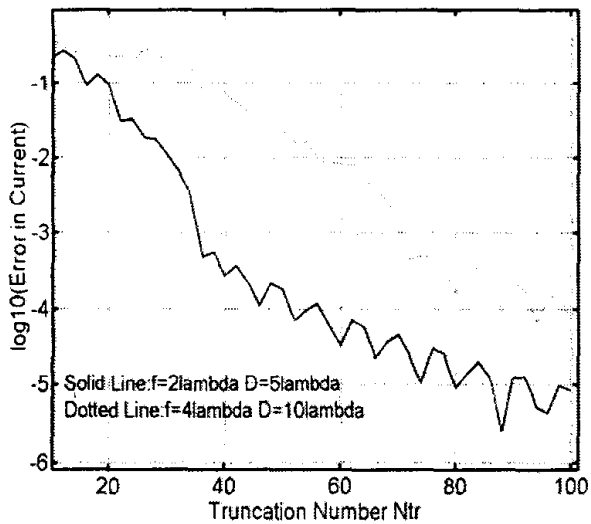

Figure 2: Truncation Error in the current coefficients. Feed directivity factor is $k b=2.6$

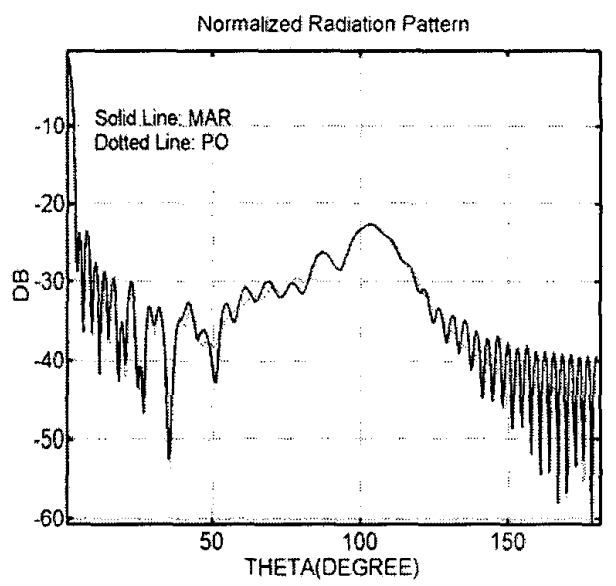

Figure 3: Comparison of radiation patterns for MAR and PO solutions. $f=8 \lambda, D=20 \lambda$. Feed directivity factor is $k b=1.5$. 